\title{
Nuclear Medicine Radiation Dosimetry: Advanced Theoretical Principles
}

\author{
B.J. McParland \\ New York, NY: Springer, 2010, 594 pages, $\$ 219$
}

This is an interesting book of 17 chapters spanning 594 pages. After the introductory chapter, chapters 2-5 describe the theoretic bases from quantum theories and Dirac's equation to radioactive decay with respect to energies and half-lives. Chapters 6-9 pertain to the interactions between radiation and matter, the different kinds of radiation, and the metrics and measurement of radiation dosimetry. Chapters 10-15 transition to the biologic effects of ionizing radiation, selected applications in nuclear medicine, anthropomorphic models, and the measurement of biodistribution in animals, with imaging, and in humans. The final 2 chapters of 10 pages conclude this book with ethical concerns and the future outlook for dosimetry. This book is well referenced and has an additional appendix of 6 pages, mostly equations for the earlier chapters.

The topics of the chapters follow a logical progression and cover the theoretic issues in radiation dosimetry in nuclear medicine with sufficient breadth. The early chapters are indeed more than adequate in the depth with which they cover essential knowledge on physics. Perhaps, as suggested by "theoretical principles" in the title, the transition to human studies, especially chapters $13-15$, is lacking in depth and examples to illustrate issues encountered in clinical radiation dosimetry.
Differential equations, vectors, and matrices are used extensively to describe the quantitative relationships of energies, phases, time, and radiation dosimetry. These conceptual and theoretic descriptions may not be readily digested by most clinicians, including nuclear physicians, radiologists, fellows, and residents. This is a book intended for physicists in nuclear medicine. The book indeed provides good theoretic backgrounds for medical physicists looking for a refresher and for physics student training in nuclear medicine. Because of the heavy theoretic overtures, it has taken me some time to read through this book. Having done that, I wish I could find more descriptions of the limitations, precision, and accuracy of radiation dosimetry in nuclear medicine and more examples on human dosimetry-topics that perhaps will have to wait for a volume on practical principles.

Franklin C. Wong

M.D. Anderson Cancer Center

1515 Holcombe Blvd., Unit 59

Houston, TX 77030

E-mail: fwong@di.mdacc.tmc.edu 